\title{
Resonancia magnética con secuencia HASTE de carcinoma epidermoide del hueso temporal
}

\author{
HASTE magnetic resonance imaging of a squamous cell temporal \\ bone carcinoma
}

Héctor Córdova J1', Alessandra Chiti $\mathbf{M}^{1}$, Natalia Cabrera $\mathrm{S}^{2}$, Carlos Stott $\mathrm{C}^{2}$, Paul H Délano $\mathrm{R}^{2}$.

\begin{abstract}
RESUMEN
El carcinoma epidermoide del hueso temporal es una neoplasia derivada de células epidérmicas del estrato espinoso, y que dado su baja incidencia, y presentación clínica similar a un cuadro de otitis media crónica colesteatomatosa, su diagnóstico es habitualmente tardío. El estudio del carcinoma epidermoide del hueso temporal se realiza con tomografía computarizada, la que evidencia erosión ósea. Sin embargo, existen distintos diagnósticos diferenciales, tanto malignos como benignos, que pueden provocar hallazgos similares en la tomografía computarizada. La resonancia magnética (RM) es un método de exploración de gran valor complementario para el estudio del carcinoma de oído medio. La RM con secuencia de difusión HASTE ha surgido como herramienta de estudio en patología de hueso temporal, tales como colesteatomas y teratomas de oído medio, pese a esto, no existen reportes en la literatura de la presentación imagenológica del carcinoma epidermoide de hueso temporal utilizando este método. En este artículo se presenta un caso clínico de una paciente con carcinoma epidermoide de oído medio, cuyo estudio preoperatorio incluyó RM-HASTE. Aun cuando se requiere un mayor número de casos para establecer el patrón imagenológico, se concluye que esta técnica es de utilidad para diferenciar este tumor de otitis media crónica colesteatomatosa.

Palabras claves: Carcinoma epidermoide de oído medio, resonancia magnética, HASTE.
\end{abstract}

\begin{abstract}
Squamous cell carcinoma of the temporal bone (SCC) is a malignancy originated from epidermis spinous cells. Its low incidence and similar presentation to cholesteatomas, contributes to its late diagnosis. The most widely used study for this tumor is computed tomography (CT), which mainly evidences erosion of the temporal bone. There are several other diagnoses, malignant and benign, that could provoke similar findings at the CT. Magnetic resonance imaging (MRI) is a method of great value to study SCC. MRI
\end{abstract}

\footnotetext{
1 Interno, Escuela de Medicina, Universidad de Chile

2 Departamento de Otorrinolaringología, Hospital Clínico Universidad de Chile.
} 
with HASTE diffusion sequence has recently emerged as an important tool in the study of temporal bone pathologies, such as cholesteatoma and middle ear teratoma, despite this, there are no reports in the literature of the imaging presentation of SCC using this method. This article presents a clinical case of a patient with SCC, in whose preoperative study was performed HASTE-MRI. Although a larger number of cases are required to establish an imaging pattern, it is concluded that this technique is useful to differentiate this tumor from middle ear cholesteatomas.

Key words: Squamous cell carcinoma, magnetic resonance imaging, HASTE.

\section{INTRODUCCIÓN}

Las lesiones neoplásicas malignas primarias del hueso temporal son entidades clínicas poco frecuentes, reportándose de 1 a 5 casos por millón de habitantes en Estados Unidos ${ }^{1-3}$. Algunas de estas neoplasias son: carcinoma de células escamosas, carcinoma de células basales, adenocarcinoma, melanoma, osteosarcoma, y linfomas entre otros $^{4-5}$, siendo el carcinoma escamoso el de mayor prevalencia, correspondiendo al $60 \%$ a $80 \%$ de los tumores de hueso temporal ${ }^{6}$.

El carcinoma epidermoide 0 de células escamosas de hueso temporal es una neoplasia derivada de células del estrato espinoso (espinocelular). Su histología se caracteriza por la presencia de nidos de células espinosas, en cuyo interior se almacena queratina, generando la imagen de "nido con perlas de queratina"1. Su incidencia es baja, reportándose anualmente sólo 2 a 4 casos por millón de habitantes ${ }^{7}$, por lo que existen pocas series de casos publicados. Se describe que este cáncer tiene un comportamiento agresivo, erosionando la pared medial del oído medio en dirección a la tuba auditiva y hacia la arteria carótida. Otra forma de extensión, es a través de la pared posterior del mastoides, alcanzando la duramadre, siendo esta forma de diseminación una causa de muerte por afección cerebral8-10.

El diagnóstico por lo general es tardío, ya que las manifestaciones tempranas son similares a la otitis media crónica colesteatomatosa, siendo los principales síntomas otorrea, otalgia e hipoacusia, mientras que la parálisis facial es un síntoma tardí0 ${ }^{11-14}$. Si bien el diagnóstico definitivo se realiza con un estudio histológico mediante biopsia ${ }^{15-16}$, el estudio imagenológico es primordial para sospechar el diagnóstico. La tomografía computarizada (TC) el examen de elección para evaluar la erosión e invasión ósea del tumor. Sin embargo, esta técnica presenta limitaciones en la evaluación de mucosa, en la evaluación del parénquima cerebral y en algunos casos es difícil detectar un tumor que no ha erosionado hueso. La resonancia magnética cerebral (RM) permite diferenciar tumor de tejido cerebral, muscular de partes blandas, vasculares y perineurales, y además puede ayudar a distinguir un tumor de cambios inflamatorios perilesionales que dificultan una adecuada delimitación del tumor ${ }^{12,17}$.

Si bien, la presencia de una masa de tejido blando en el interior del canal auditivo, asociado a erosión ósea son algunos de los principales hallazgos encontrados en la exploración imagenológica de este tumor, existen distintos diagnósticos diferenciales tanto malignos como benignos que pudiesen provocar estos mismos hallazgos. Dentro de éstos encontramos distintas entidades que comprometen el oído medio, como enfermedades granulomatosas y procesos inflamatorios como el colesteatoma, siendo este último uno de los principales diagnósticos diferenciales en el reconocimiento de este tumor ${ }^{18-21}$. Es por esto, que si bien la TC es el examen primordial para detectar el carcinoma escamoso de hueso temporal, la RM es un examen complementario de gran valor diagnóstico, ya que permite realizar diagnóstico diferencial con procesos inflamatorios de tejidos blandos.

Pese a que en conjunto la TC y la RM constituyen el estudio fundamental para realizar el diagnóstico de este tumor, no presenta exacta correlación con los hallazgos intraoperatorios. Se ha descrito en la literatura que en conjunto la TC y RM presentan precisión global de sólo $85 \%$ en relación a los hallazgos intraoperatorios ${ }^{22}$, mientras que lesiones de menos de $2 \mathrm{~mm}$ encontradas en las biopsias no se lograron visualizar en la $\mathrm{TC}^{23}$.

En relación a esto, durante los últimos años se han descrito nuevos métodos diagnósticos en 
procesos patológicos ubicados en hueso temporal. En este contexto, la resonancia magnética ponderada por difusión (DW-MRI) ha adquirido un papel creciente en el diagnóstico de patología de hueso temporal, como los colesteatomas de oído medio; basándose en el patrón restrictivo producido por el contenido epitelial queratinizado. El DW-MRI convencional ecoplanar (EPI) ha sido reemplazado por secuencias no-EPI DW-MRI porque minimizan los artefactos de susceptibilidad en la base del cráneo y aumentan la sensibilidad para la detección de colesteatomas tan pequeños como $2 \mathrm{~mm}^{24-26}$. En relación a las técnicas no-EPI DW-MRI la secuencia más utilizada es la Half-Fouríer acquired singleshot turbo spin echo (HASTE), que corresponde a una secuencia no-EPI DW-MRI abreviada en T227. Con el uso de este método se ha informado una alta sensibilidad y especificidad ( $>90 \%$ ) para el diagnóstico de colesteatomas primarios y secundarios con el protocolo HASTE ${ }^{26,28-31}$. En relación a tumores del oído medio, se ha descrito la presencia de un patrón granular en la RM HASTE de teratomas del oído medio ${ }^{28}$. No existe reporte en la literatura sobre las características imagenológicas del carcinoma epidermoide de oído medio con RM HASTE.

A continuación se presenta un caso clínico correspondiente a una paciente atendida en el Hospital Clínico de la Universidad de Chile ( $\mathrm{HCUCH})$ con sospecha clínica de carcinoma epidermoide de hueso temporal, en quien se realizó RM HASTE y cirugía posterior con toma de biopsias.

\section{CASO CLÍNICO}

Paciente de sexo femenino, 52 años que consulta en HCUCH en marzo de 2015 por cuadro de 3 meses de evolución de otorrea y otalgia de oído izquierdo (이) asociado a cefalea holocránea, sin otros síntomas acompañantes. Al examen físico destacaba en la otomicroscopía de oído izquierdo un aumento de volumen en pared posterior sensible a la palpación y laminillas de piel en CAE las cuales se aspiran sin lograr visualizar tímpano. En oído derecho, sólo se observaba miringoesclerosis. En este contexto se solicitan exámenes donde destacaba: Audiometría: Hipoacusia de conducción en oído izquierdo con PTP 0I: 40/10 dB, discriminación Ol: a $60 \mathrm{~dB}$ $100 \%$. Sin alteraciones en oído derecho (PTP aéreo $5 \mathrm{~dB}$ ). La TC de oídos sin contraste muestra en OI ocupación de oído medio y mastoides con densidad de partes blandas, con dehiscencia hacia fosa posterior (Figura 1). La RM de cerebro muestra una imagen hipointensa en T2 (Figura 2A), mientras que en la secuencia $\mathrm{T1}$ con gadolinio (Figura 2B) se observa captación periférica de la lesión. En relación a la secuencia de difusión HASTE, no se observa restricción a la difusión en caja timpánica (Figura 2C).

Se planificó cirugía radical OI con toma de biopsia por tumor de oído medio izquierdo. Al realizar la mastoidectomía se evidencia tejido epitelial y granuIomatoso en mastoides, con dehiscencia amplia hacia seno lateral y fosa posterior, además se observa meninges de fosa posterior con infiltración tumoral,

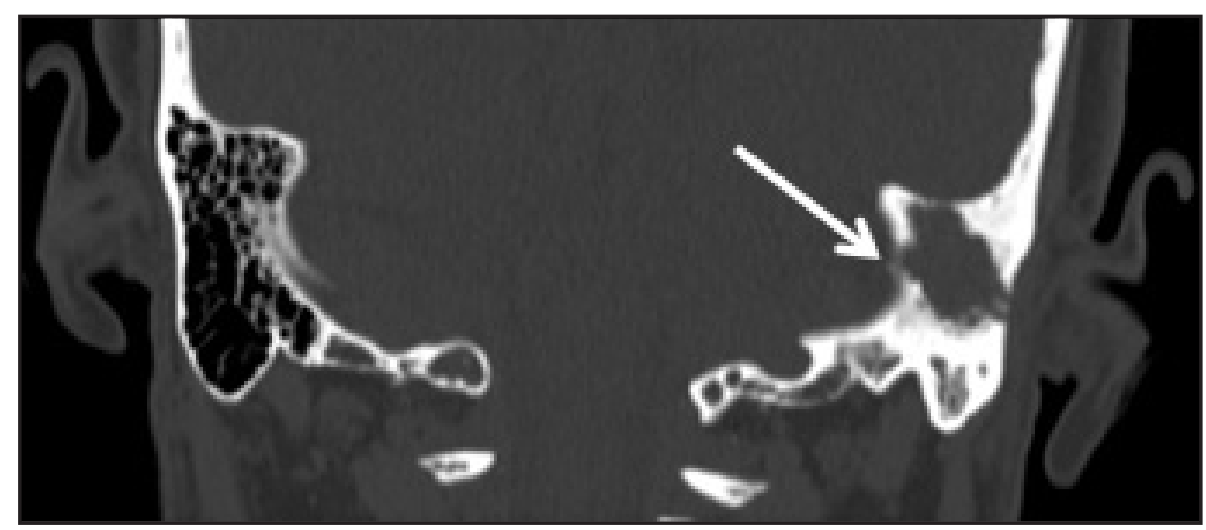

Figura 1. 


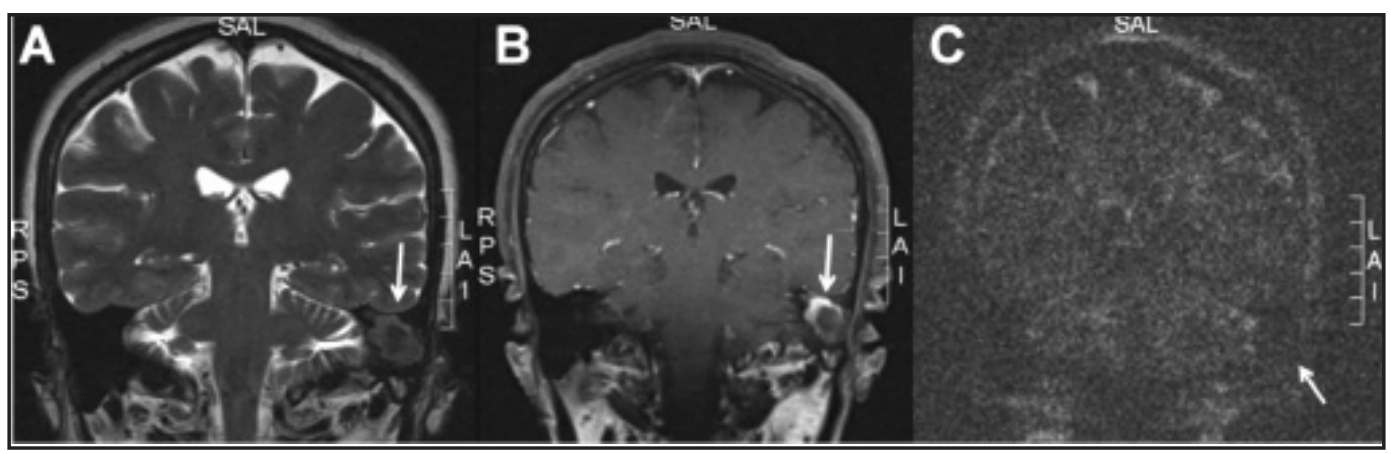

Figura 2

sin fístula de líquido cerebroespinal. Biopsia diferida informa epitelio plano pluriestratificado con hiperqueratosis y presencia de remolinos córneos, hiperplasia pseudoepiteliomatosa e infiltración del estroma subyacente. Histopatología compatible con carcinoma epidermoide bien diferenciado infiltrante. El Comité Oncológico del Hospital de la Universidad de Chile decide realizar radioterapia paliativa debido al compromiso de la duramadre del cerebelo.

\section{DISCUSIÓN}

El carcinoma epidermoide del hueso temporal es una entidad de baja incidencia ${ }^{6-7}$ pero de comportamiento sumamente agresivo, lo que genera la necesidad de encontrar métodos diagnósticos que permitan sospechar esta patología. En 2013 Zhang y cols. realizaron una descripción de 12 casos, en el que se reportan características imagenológicas de este tumor en TC y RM ${ }^{17}$. En la TC se describe la presencia de una masa de tejido blando localizada en hipotímpano, fosa yugular y canal auditivo externo, que se asocia a erosión y destrucción ósea, siendo el compromiso óseo lo que se presenta más claramente en este examen $^{16,18,32}$; lo que es concordante con lo encontrado en el TC de esta paciente (Figura 1). Sin embargo la erosión ósea también puede aparecer en TC de pacientes con sospecha de colesteatoma.

En los últimos años la RM con secuencia HASTE ha surgido como una técnica diagnóstica sensible $(82 \%-100 \%)$ y específica para colesteatoma ${ }^{26,29,33-35}$, siendo incluso utilizado en nuestro medio como técnica de detección de colesteatoma residual 0 recurrente en pacientes con cirugía previa de oído medio ${ }^{26}$. En la evaluación con RM HASTE, Ios colesteatomas presentan restricción de la señal, lo que se observa como una imagen blanca intensa en la secuencia ${ }^{26-30}$. Por el contrario y a pesar de la presencia de una lesión con captación periférica de gadolinio en la secuencia T1 de la RM (que es lo que se describe en colesteatomas), la paciente presentada en este caso clínico no demostró aumento de la intensidad de señal (restricción de difusión) a nivel de la mastoides izquierda en la secuencia de difusión RM HASTE. De esta forma, el diagnóstico de colesteatoma queda prácticamente descartado con la RM HASTE. Es importante notar que este caso clínico es el primer reporte en la literatura acerca de la imagen radiológica generada por el carcinoma epidermoide en la resonancia magnética con protocolo de difusión HASTE, por lo que no teníamos antecedentes de si las células epidermoides del tumor producirían 0 no una señal de restricción en la RM HASTE.

\section{CONCLUSIÓN}

La RM HASTE es una herramienta diagnóstica relativamente reciente que está adquiriendo importancia en el diagnóstico diferencial de masas tumorales del oído medio. En este artículo se muestra una imagen de un carcinoma epidermoide de hueso temporal con secuencia de difusión RM HASTE negativa, ayudando a diferenciarlo de otras entidades clínicas como colesteatomas o teratomas. 


\section{BIBLIOGRAFÍA}

1. GidLey PW, Roberts DB, Sturgis EM. Squamous cell carcinoma of the temporal bone. Laryngoscope 2010; 120: 1144-51.

2. Kuhel WI, Hume CR, Selesnick SH. Cancer of the external auditory canal and temporal bone. Otolaryngol Clin North Am 1996; 29: 827-52.

3. BARRS DM. Temporal bone carcinoma. Otolaryngol Clin North Am 2001; 34: 1197-218.

4. Bacciu A, Clemente IA, Piccirillo E, et al. Guidelines for treating temporal bone carcinoma based on long-term outcomes. Otol Neurotol 2013; 34: 898-907.

5. Prasad SC, D'Orazio F, Medina M, Bacciu A, Sanna M. State of the art in temporal bone malignancies. Curr Opin Otolaryngol Head Neck Surg 2014; 22: 154-65.

6. Moffat DA, Wagstaff SA. Squamous cell carcinoma of the temporal bone. Curr Opin Otolaryngol Head Neck Surg 2003; 11: 107-11.

7. Yadav S, Gupta D, Yadav GS. Squamous cell carcinoma of the external auditory canal in young: A rare case report. Indian Journal of Otology 2013; 19: 196-8.

8. Beyea JA, Moberly AC. Squamous Cell Carcinoma of the Temporal Bone. Otolaryngol Clin North Am 2015; 48: 281-92.

9. Shu MT, Lee JC, Yang CC, Wu KC. Squamous cell carcinoma of the middle ear. Ear, Nose and Throat Journal 2012; 91: 14-5.

10. LEwIS JS. Temporal bone resection: review of 100 cases. Arch Otolaryngol 1975; 101: 23-5.

11. Hu XD, Wu TT, Zhou SH. Squamous cell carcinoma of the middle ear: report of three cases. Int J Clin Exp Med 2015; 8: 2979-84.

12. Gillespie MB, Francis HW, Chee N, Eisele DW. Squamous cell carcinoma of the temporal bone: a radiographic-pathologic correlation. Arch Otolaryngol Head Neck Surg 2001; 127: 803-7.

13. NoorizAn Y, Asma A. Temporal bone carcinoma: a case report. Med J Malaysia 2010; 65: 162-4.

14. Leong S, Youssef A, Lesser TH. Squamous Cell Carcinoma of the Temporal Bone: Outcomes of Radical Surgery and Postoperative Radiotherapy. Laryngoscope 2013; 123: 2442-8.

15. Zhang B, Tu G, Xu G, Tang $P$, Hu Y. Squamous cell carcinoma of temporal bone: reported on 33 patients. Head Neck 1999; 21: 461-6.
16. Michaels L, Wells M. Squamous cell carcinoma of the middle ear. Clin Otolaryngol Allied Sci 1980; 5: 235-48.

17. Zhang F, Sha Y. Computed tomography and magnetic resonance imaging findings for primary middle-ear carcinoma. J Laryngol Otol 2013; 127: 578-83.

18. Juliano AF, Ginat DT, Moonis G. Imaging review of the temporal bone: part I. Anatomy and inflammatory and neoplastic processes. Radiology 2013; 269: 17-33.

19. Heilbrun ME, Salzman KL, Glastonbury CM, Harnsberger HR, Kennedy RJ, Shelton C. External auditory canal cholesteatoma: clinical and imaging spectrum. AJNR Am J Neuroradiol 2003; 24: 751-6.

20. Aswani Y, Varma R, Achuthan G. Spontaneous external auditory canal cholesteatoma in a young male: Imaging findings and differential diagnoses. Indian J Radiol Imaging 2016; 26 : 237-40.

21. Tomazic PV, Ropposch T, Karpf EF, Nemetz U, Braun EM, Lackner A, Walch C. Giant Middle Ear Squamous Cell Carcinoma Mimicking as Cholesteatoma. Otol Neurotol 2012; 33: e39-e40.

22. LeONetTI JP, SMith PG, KLetzKer GR, IZQuierdo R. Invasion patterns of advanced temporal bone malignancies. Am J Otol 1996; 17: 438-42.

23. Hosokawa S, Mizuta K, Takahashi G, Okamura J, Takizawa Y, Hosokawa K, Mineta H. Surgical approach for treatment of carcinoma of the anterior wall of the external auditory canal. Otol Neurotol 2012; 33: 450-4.

24. De Foer B, Vercruysse, JP, Spaepen, M, Somers, T, Pouillon, M, Offeciers, E, Casselman, JW. Diffusion-weighted magnetic resonance imaging of the temporal bone. Neuroradiology 2010; 52 : 785-807.

25. Moura M, Taranto, D, García, M. Cholesteatoma: utility of non-echo-planar diffusion-weighted imaging. Radiol Bras 2012; 45: 283-7.

26. Cabrera N, Sedano C, Delano P, Alvo A. Uso de resonancia magnética con secuencia de difusión no-ecoplanar para la detección de colesteatoma en pacientes con cirugía de oído previa: Presentación de 4 casos. Rev Otorrinolaringol Cir Cabeza Cuello 2015; 75: 145-55.

27. Patel MR, Klufas Ra, Alberico Ra, Edelman RR. Half-fourier acquisition single-shot turbo spin- 
echo (HASTE) MR: comparison with fast spinecho MR in diseases of the brain. AJNR Am J Neuroradiol 1997; 18: 1635-40.

28. León F, Alvo A, Sanhueza D, Delano PH, Stott CE. HASTE Diffusion-Weighted Magnetic Resonance Imaging of Middle Ear Teratoma. Otol Neurotol 2015; 36: e156-e158.

29. Li PM, Linos E, Gurgel RK, Fischbein NJ, Blevins $\mathrm{NH}$. Evaluating the utility of non-echo-planar diffusion-weighted imaging in the preoperative evaluation of cholesteatoma: a meta-analysis. Laryngoscope 2013; 123: 1247-50.

30. Alvo A, Garrido C, Salas Á, Miranda G, Stott CE, Delano PH. Use of non-echo-planar diffusionweighted MR imaging for the detection of cholesteatomas in high-risk tympanic retraction pockets. AJNR Am J Neuroradiol 2014; 35: 1820-4.

31. Alzérreca E, Zamorano R, Salas A, Délano PH. Resonancia magnética cerebral con secuencia difusión - HASTE en la evaluación clínica del colesteatoma. Rev Otorrinolaringol Cir Cabeza Cuello 2011; 71: 249-56.

32. Bird CR, Hasso AN, Stewart CE, Hinshaw JR DB, THOMPSON JR. Malignant primary neoplasms of the ear and temporal bone studied by highresolution computed tomography. Radiology 1983; 149: 171-4.

33. Más-Estellés F, Mateos-Fernández, M, CrrascosaBisquert, B, Facal de Castro, F, Puchades-Román, I, Morera-Pérez, C. Contemporary non-echoplanar diffusion-weighted imaging of middle ear cholesteatomas. Radiographics 2012; 32: 1197-213.

34. Sharifian H. Diagnostic accuracy of non-echoplanar diffusion-weighted MRI versus other MRI sequences in cholesteatoma. $J$ Med Imaging Radiat Oncol 2012; 56: 398-408.

35. Plouin-Gaudon I, Bossard D, Ayari-Khalfallah $S$, Froehlich P. Fusion of MRIs and CT scans for surgical treatment of cholesteatoma of the middle ear in children. Arch Otolaryngol Head Neck Surg 2010; 136: 878-83. 